\title{
STAR FORMATION
}




\title{
MOLECULES IN STAR FORMATION
}

\author{
F.H. SHU \\ Astronomy Department, University of California \\ Berkeley, CA 94720-3411, USA
}

\begin{abstract}
We review current ideas and models in the problem of star formation from molecular cloud cores that are relatively isolated from the influences of other forming stars. We discuss the time scales, flow dynamics, and density and temperature structures applicable to each of the four stages of the entire process: (a) formation of a magnetized cloud core by ambipolar diffusion and evolution to a pivotal state of gravomagneto catastrophe; (b) self-similar collapse of the pivotal configuration and the formation of protostars, disks, and pseudo-disks; (c) onset of a magnetocentrifugally driven, lightly ionized wind from the interaction of an accretion disk and the magnetosphere of the central star, and the driving of bipolar molecular outflows; (d) evolution of pre-main-sequnce stars surrounded by dusty accretion disks. For each of these stages and processes, we consider the characteristics of the molecular diagnostics needed to investigate the crucial aspects of the observational problem.
\end{abstract}

\section{Introduction}

My task is to provide a theoretical setting for the role of molecules in star formation. Astronomers lack a complete analysis for the full problem of star formation. I shall therefore confine my remarks to the simplest and best studied case - the formation of low-mass stars by an isolated mode in an environment like the Taurus molecular cloud (Myers 1995). Star formation by the cluster mode is also an important process (Lada et al. 1991, Lada 1992), but a generally accepted description of this case does not yet exist.

In contrast, over the past decade, spurred by the rapid accumulation of observational data of unprecedented quality and resolution over a broad spectral range (see the review of Blake in this volume), astronomers have been able to piece together a reasonably successful picture for the formation 

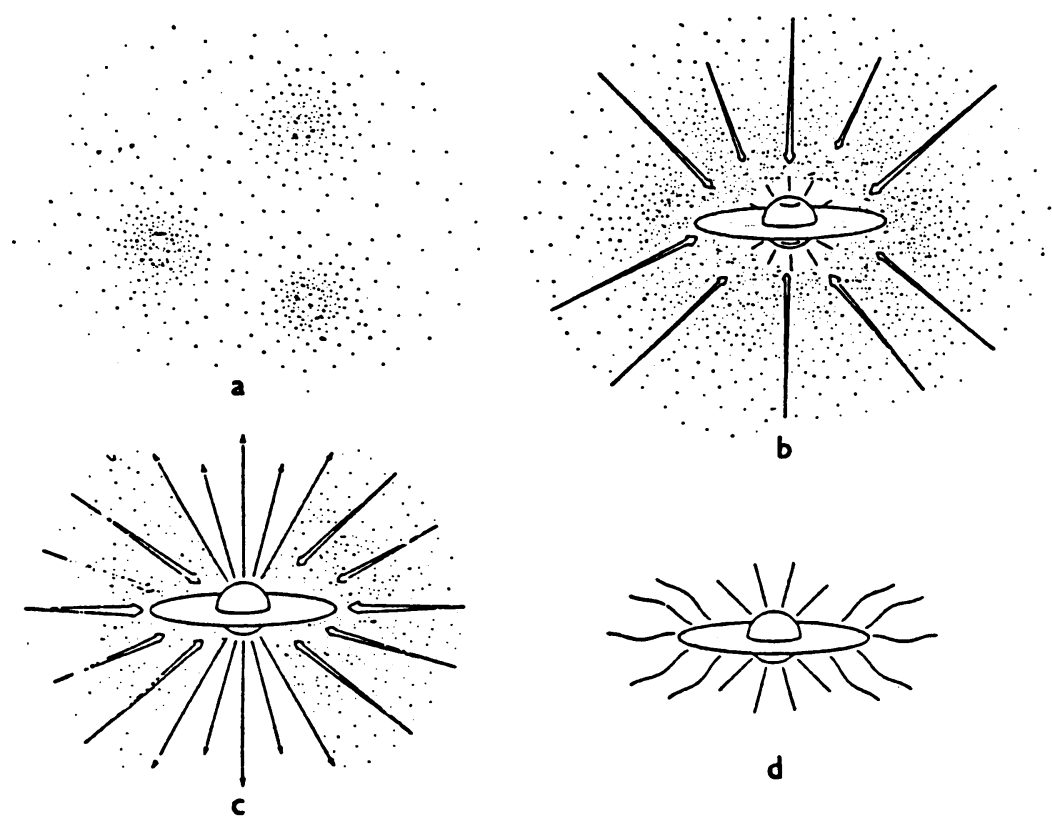

Figure 1. The four stages of low-mass star-formation in the isolated mode (from Shu, Adams \& Lizano 1987). (a) Molecular cloud cores form via the diffusive leakage of magnetic fields from the dense neutral concentrations. (b) The inside-out collapse of a slowly rotating and weakly magnetized core leads to the formation of a protostar and a disk (plus perhaps an infalling "pseudodisk" in the equatorial directions). (c) A collimated wind breaks out along the rotational poles, creating a bipolar outflow in the surrounding molecular material. (d) The dusty infall from the cloud core is reversed over all solid angles by the outflow, revealing a newly born star surrounded by an accretion disk.

of isolated low-mass stars (see, e.g., the review of Evans 1991). The main processes can be usefully divided into four schematic stages (see Fig. 1), the details of which will be described in the sections below.

\section{The formation of molecular cloud cores}

In the first stage $(t<0)$, dense pockets of gas and dust contract quasistatically by the mechanism of ambipolar diffusion from a rarefied, turbulent, 
and magnetized common envelope that characterizes the rest of the molecular cloud (Nakano 1979, Lizano \& Shu 1989, Fiedler \& Mouschovias 1993, Basu \& Mouschovias 1994). The ratio of the diffusive evolutionary timescale to the gravitational free-fall timescale is given by the dimensionless coupling parameter for ion-neutral collisions (see Shu 1983, Galli \& Shu 1993):

$$
\Gamma \equiv \frac{\gamma C}{(4 \pi G)^{1 / 2}}
$$

In equation (1), $G$ is the universal gravitational constant, $\gamma \equiv\langle w \sigma\rangle /(m+$ $\left.m_{\mathrm{i}}\right)$ is the frictional drag coefficient associated with collisions at a mean rate $\langle w \sigma\rangle$ between ions of mass $m_{\mathrm{i}}$ and neutrals of mass $m$, and $C$ is the coefficient in the relation $\rho_{\mathrm{i}}=C \rho^{1 / 2}$ between ion mass density $\rho_{\mathrm{i}}$ and neutral mass density $\rho$ when ionization by Galactic cosmic rays are balanced by dissociative recombinations. For standard values of $\gamma$ (Draine et al. 1983) and $C$ (Nakano \& Umebayashi 1980; see also de Boisanger et al. 1996), $\Gamma=11$, giving an ambipolar diffusion timescale typically of a few million years to form dense molecular cloud cores of the type observed in $\mathrm{NH}_{3}$ by Myers \& Benson (1983) and in ${ }^{13} \mathrm{CO}$ and $\mathrm{C}^{18} \mathrm{O}$ by Mizuno et al. (1994).

No appreciable spinup occurs during the formation of the molecular cloud core, because torsional Alfven waves have sufficient time to keep the contracting core rotating roughly at the same angular speed as the surrounding envelope (Mestel \& Paris 1979, Mouschovias 1979). As the central region of a potential star-forming site passes beyond a critical mass-to-flux ratio, the molecular cloud core undergoes a gravomagneto catastrophe that leads to the formation of a magnetized, slowly rotating, singular isothermal toroid (Li \& Shu 1996). Ideally, this axisymmetric configuration has density and magnetic flux distributions in spherical polar coordinates of the forms,

$$
\rho(r, \theta)=\frac{a^{2} R(\theta)}{2 \pi G r^{2}}, \quad \Phi(r, \theta)=\frac{4 \pi a^{2} r \phi(\theta)}{G^{1 / 2}},
$$

where $a=(k T / m)^{1 / 2}$ is the isothermal sound speed of the pre-collapse medium at temperature $T$. In equation $(2), R(\theta)$ and $\phi(\theta)$ are dimensionless functions whose forms depend on the amount of relative support $H_{0}$ provided by the magnetic field compared to thermal pressure at the instant $(t=0)$ of gravomagneto catastrophe.

Li \& Shu (1996) estimate that $H_{0} \approx 0.5$ to reproduce the average flattening of Taurus molecular cloud cores observed by Myers et al. (1991). For a gas kinetic temperature $T=10 \mathrm{~K}, a=0.2 \mathrm{~km} \mathrm{~s}^{-1}$, and equation (2) then implies that $\rho=1 \times 10^{-19} \mathrm{~g} \mathrm{~cm}^{-3}$ and $B=15 \mu \mathrm{G}$ at an equatorial distance $r=0.05 \mathrm{pc}$ from the center of a molecular cloud core, in good agreement with the measurements of Benson \& Myers (1989) and Crutcher et al. (1993). 


\section{Self-similar gravitational collapse}

The subsequent dynamical collapse $(t>0)$ can be studied semi-analytically by similarity techniques, and leads to the second stage, whereby a star plus centrifugally supported disk accretes matter from an infalling envelope at a uniform rate,

$$
\dot{M}=\mathcal{M}_{0}\left(1+H_{0}\right) a^{3} / G,
$$

where $\mathcal{M}_{0}=0.975$ for the case $\left(H_{0}=0\right)$ of the singular isothermal sphere (Shu 1977) and $\mathcal{M}_{0}=1.05$ for the case $\left(H_{0} \rightarrow \infty\right)$ of the singular isothermal disk (Li \& Shu 1997). For $H_{0}=0.5$ and $a=0.2 \mathrm{~km} \mathrm{~s}^{-1}$, equation (3) yields $\dot{M}=3 \times 10^{-6} M_{\odot} \mathrm{yr}^{-1}$, which is in approximate agreement with the average infall rate $\dot{M}=4 \times 10^{-6} M_{\odot} \mathrm{yr}^{-1}$ deduced by Kenyon et al. (1993) from infrared spectral-energy modeling of protostars with infalling envelopes. At such a rate, it takes a few hundred thousand years to accumulate a sunlike star, in reasonable accord with the observation that embedded protostars in Taurus are an order of magnitude less common than revealed pre-mainsequence stars ( $\mathrm{T}$ Tauri stars with an average age of probably a few million years; see Cohen \& Kuhi 1979, Strom et al. 1989).

In all cases, the theory predicts the gravitational collapse to occur characteristically from inside-out, with the largest infall velocities occurring at the smallest radii. Since the collisional excitation of molecules is also stronger at smaller $r$ where the density is higher, inside-out collapse models predict asymmetric line-profiles for molecular transitions that are not optically thin, with the emission in the redshifted portion of the line wings being weaker because of self-absorbtion than the blueshifted portion. Asymmetries of the correct qualitative and quantitative form have been found and modeled for $\mathrm{CS}$ and $\mathrm{H}_{2} \mathrm{CO}$ in the B335 region of low-mass star formation by Zhou et al. (1993) and Choi et al. (1995).

In the presence of dynamically important levels of the magnetic field, infall occurs into a pseudodisk whose radius grows with time $t$ but is typically of order $10^{3} \mathrm{AU}$ (Galli \& Shu 1993). The latter value, as well as details of the predicted velocity and density fields are in reasonable accord with the ${ }^{13} \mathrm{CO}$ measurements of a flattened infalling envelope around the protostellar source HL Tau (Hayashi et al. 1993).

If the original molecular cloud spins at a rotational speed that is only a small fraction of the sound speed $a$, material in the pseudodisk is not in mechanical equilibrium. This gas continues to flow inward at magnetically diluted free-fall speeds until it reaches a centrifugal disk whose radius also grows with time $t$ but is typically of order $10^{2}$ AU (Cassen \& Moosman 1981, Terebey et al. 1984, Li \& Shu 1997). At radio wavelengths, such true disks may be observationally studied both in the thermal continuum and in selected molecular lines (see the review by Blake in this volume). Generally, 
such studies yield disk sizes and orientations (with respect to the outflow axes) that are in good agreement with theoretical expectations.

Inside the centrifugal disk, mechanisms must exist (Lin \& Papaloizou 1993) that transport angular momentum outwards and mass inwards, feeding the growth of an ever more massive protostar. The most promising anomalous viscosity mechanism is the so-called Balbus-Hawley (1991) instability. However, the midplane densities are very high even if the disks surrounding young stellar objects contain only the minimum mass needed to create the planets of the solar system (Hayashi et al. 1985). The gases in the interiors of such disks are so well-shielded from external sources of ionizing radiation at radii from about $0.1 \mathrm{AU}$ to $10 \mathrm{AU}$ that they may be effectively decoupled from magnetic fields (Nakano \& Umebayashi 1980). Thus, the Balbus-Hawley instability may operate only in the surface layers of such disks (Gammie 1996, see also the contribution of Glassgold in this volume), greatly reducing the efficiency of the viscous transport mechanism compared to conventional estimates.

Empirically, the disk accretion rate $\dot{M}_{D}$ probably equals the infall rate $\mathcal{M}_{0}\left(1+H_{0}\right) a^{3} / G$ only on average. The instantaneous rate (at least the part that reaches a few stellar radii) may exhibit high states (outbursts) and low states (quiescence) (see, e.g., Hartmann et al. 1993; Bell \& Lin 1994). Gravitational instabilities of a spiral or bar nature may drive the early evolution of the disks (e.g., Laughlin \& Bodenheimer 1993). It is not known whether such instabilities merely promote the general phenomenon of the outward transport of angular momentum and the inward transport of mass, or whether they can also lead to disk fragmentation and the formation of binary and multiple star systems. In any case, spiral density waves driven in the disk by resonant gravitational interaction with a nearby orbiting body can also contribute to disk transport (Ostriker et al. 1992, Yuan \& Cassen 1994).

\section{Winds, jets, and bipolar molecular outflows}

The third stage begins when deuterium burning in the growing, rotating, protostar creates an outer convection zone (Stahler et al. 1980, Palla \& Stahler 1992), allowing dynamo-generated magnetic fields to rise to the surface and initiate the measurable phenomena (e.g., flares and X-ray emission) that we associate with stellar magnetospheres (Shu \& Terebey 1984). The interaction of the stellar magnetosphere with the surrounding accretion disk locks the rotation rate of the central star to that of the inner edge of a truncated accretion disk (Königl 1992, Shu et al. 1994). It also drives a magnetocentrifugal wind from the same inner regions of the disk (Arons 1986, Shu et al. 1988, Camenzind 1990, Najita \& Shu 1994). 
The detailed theory of this process yields a rate of wind outflow $\dot{M}_{\mathrm{w}}$ directly proportional to the rate of disk inflow $\dot{M}_{D}$, i.e., $\dot{M}_{\mathrm{w}}=f \dot{M}_{D}$, with the fraction $f$ estimated as $\sim 1 / 3$ by the "preferred model" of Najita \& Shu (1994) and Ostriker \& Shu (1995). Such a high value for $f$ is compatible with indirect estimates for the momentum requirements of the drivers of bipolar molecular outflows (Lada 1995), but it is higher than direct estimates (Hartigan et al. 1995) for $\dot{M}_{\mathrm{w}} / \dot{M}_{D}$ obtained from the ratio of the mass loss rate carried in optical jets to the mass accretion rate onto the central star. The latter is deduced from the level of optical "veiling" present, i.e., the amount by which the strength of photospheric lines is reduced in comparison with the continuum radiation. The direct method carries considerable uncertainty concerning the fractional ionization in (inhomogeneous) stellar jets.

Far from the star, the outflow self-collimates into jets surrounded by a wide-angle wind (Shu et al. 1995, Li 1996). At distances greater than $\sim 10$ $\mathrm{AU}$, the density acquires a cylindrically-stratified wind profile,

$$
\rho_{\mathrm{w}} \propto \frac{1}{r^{2} \sin ^{2} \theta},
$$

with a velocity profile that is much less sensitive to variations with $\theta$. Shu et al. (1991; see also Masson \& Chernin 1992) propose a simplified model for bipolar molecular outflows based on the interaction of a protostellar wind (or jet) with power dependent on polar angle $\theta$ as $P(\theta)$, flowing into a molecular cloud core with a density profile given by $\rho(r, \theta)=a^{2} R(\theta) / 2 \pi G r^{2}$ [see eq. (2)]. Li \& Shu (1996) show that the choice $P(\theta) \propto 1 / \sin ^{2} \theta$ combined with the $R(\theta)$ appropriate for a singular molecular cloud core with $H_{0}=0.5$ yields (a) shapes of swept-up molecular lobes, (b) integrated line profiles, (c) distributions of swept-up mass at different line-of-sight velocities, and (d) distributions of momenta at different projected distances along the bipolar flow axes that agree well with the observations (Masson \& Chernin 1992, 1993; Chernin \& Masson 1995; Lada \& Fich 1996).

The outflow initially breaks through the surrounding envelope only near the rotational poles. Rotating infall still occurs in the equatorial regions; thus, the third stage is one of simultaneous inflow and outflow. With infall occurring in the presence of a much stronger outflow, it is perhaps not surprising that the best cases for the detection of the inflow are sources like B335 where the outflow occurs in the plane of the sky and therefore has a relatively low component of velocity along the line of sight (Zhou et al. 1993). The quantitative models of bipolar outflow interaction reviewed above offer a promising theoretical method to expand the range of sources that can be analyzed for equatorial infall. Such methods will be especially powerful if they can be combined with chemical diagnostics that separate 
inflowing and outflowing material. The observations of van Langevelde et al. (1994) and Blake et al. (1994) suggest, respectively, that the molecules $\mathrm{HCO}^{+}$and $\mathrm{SiO}$ may serve these diagnostic functions in low-mass starforming regions (as they do in high-mass star-forming regions; see Welch et al. 1987; Wright et al. 1996).

Early in the third stage of star formation (see Fig. 1) when the infall occurs close to the central object and the outflow is highly collimated, the infrared spectral energy distribution (SED) would have the appearance of a Class 0 source (André et al. 1993). Later, when the column densities decline somewhat, an external observer located away from the rotational poles would measure an SED typical of a Class I source (reprocessed farinfrared radiation from the infalling envelope plus, perhaps, some scattered near-infrared radiation from the polar holes; see Whitney \& Hartmann 1993); whereas an observer nearly aligned with the polar direction may measure something akin to a "flat-spectrum T Tauri star" or a "doublehumped SED" (transition cases between a Class I and Class II source; see Adams et al. 1987).

\section{T Tauri stars and their accretion disks}

The fourth stage begins when, for incompletely understood reasons, the outflow widens and reverses the inflow over essentially all $4 \pi$ steradians, revealing the central star and its accretion disk to all optical and nearinfrared observers (expect possibly those who happen to lie nearly exactly in the disk midplane). The measured SED would contain the considerable near- and mid-infrared excess from the disk, which characterizes a Class II source (Lynden-Bell \& Pringle 1974, Rucinski 1985; Kenyon \& Hartmann 1987, Adams et al. 1988, Beckwith et al. 1988, Strom et al. 1989). Because of the presence of an inner accretion disk and the interactions it induces, the central object would be classified as a classical T Tauri star (see the review of Bertout 1989). The smaller $\dot{M}_{D}$ yields a lower-rate of mass-loss $\dot{M}_{\mathrm{w}}=$ $f \dot{M}_{D}$ carried in the magnetocentrifugally driven wind. The corresponding magnetospheric inflow $\dot{M}_{*}=(1-f) \dot{M}_{D}$ onto the star gives an accretion funnel that impacts the mid-latitudes of the star at near-free-fall speeds (Bertout et al. 1988, Hartmann et al. 1994, Ostriker \& Shu 1995), releasing the energetic photons from hot spots that may account for the optical veiling and ultraviolet excessses associated with classical $\mathrm{T}$ Tauri stars (see the review of Bertout 1989).

Heating of the disk's surface layers by radiation from the central star can invert the normal vertical temperature distribution that we expect for an accretion disk, putting $\mathrm{CO}$ rotational-vibrational transitions into emission rather than absorption (Calvet et al. 1991). Carr et al. (1993) and 
Najita et al. (1996) have analyzed the CO overtone emission line-profiles observed in the sources WL 16 and 1548C27. These analyses yield some of the most definitive evidence that gaseous Keplerian disks exist around some young stellar objects that extend nearly to the surface of the central stars. Good diagnostics await to be developed for measuring the gas-todust content of such disks. Until then, we only have qualitative estimates for the timescales over which the solid materials in the disk are exhausted by accumulation in planetary (or stellar) companions, and over which gas in the disk is lost by accretion onto the central star (or giant planets), or by dispersal through the action of $\mathrm{T}$ Tauri winds, or by photoevaporation via ultraviolet photons. The disappearance of the dust in the disk will convert a Class II SED to a Class III SED, typical of bare stars. On the other hand, when the nebular gas in the surface layers of the disk, wind, and funnel flow disappear, the emission-line characteristics will weaken, leading the central pre-main-sequence star to be classified as a weak-lined $\mathrm{T}$ Tauri star. Magnetospheric activity will continue to exist as long as the star continues to rotate appreciably and to possess an outer convection zone (Walter 1986), so young stars may continue to emit X-rays even when they have contracted to the main sequence (Briceño et al. 1997).

\section{Summary and prospectus}

Star formation affords a premier example of an astronomical field where molecules play crucial roles in important physical processes. Molecules provide not only the principal coolants for molecular cloud cores, determining thereby the crucial parameter $a$ that enters so prominently in much of our above discussions, but their ions also carry most of the electrical charge in the system until we get to very high densities (above $10^{11} \mathrm{~cm}^{-3}$ according to Nakano \& Umebayashi 1980). This ionization level then sets the timescale for ambipolar diffusion to produce dense molecular cloud cores. McKee (1989) has proposed that the slippage is self-regulated and provides the rate-limiting factor for low-mass star formation in giant molecular clouds. Because the molecular ions determine the degree of dynamical coupling to ambient magnetic fields, they may also control the magnetic flux loss from protostars and their pseudodisks, and they may dictate how angular momentum and mass transport occur in the centrifugally supported disks surrounding young stellar objects.

Molecules also constitute the main diagnostics by which we observationally infer the density, velocity, and temperature distributions in dynamically evolving protostellar regions. Here, the comparable order of magnitudes for the timescales of the magnetohydrodynamics and of molecule formation and destruction (or deposition onto grain surfaces) yield both opportuni- 
ties and challenges. The opportunities come from the possibility of using the molecules as chemical clocks of the dynamics. Alternatively, the theoretically computed dynamics may serve as a calibrator of competing chemical pathways. The challenges arise because the simplest comparisons between theory and observation often assume constant abundances for the tracer species. In reality, the total problem may be a complex one with nonlinear feedbacks between the magnetohydrodynamics, chemical transformations, and the energetics of the radiative transfer. To make additional progress in the future will probably require an iterative approach. To succeed, it will be important have good starting guesses. Toward this end, I formulate below what I consider are the crucial semi-empirical questions that need answering at the next molecules conference:

- What are the (nearly) constant abundance species in molecular cloud cores? (Dust? CO? CS?)

- What molecules are good tracers for gravitational collapse? $\left(\mathrm{HCO}^{+}\right.$? $\mathrm{CS}$ ? $\mathrm{H}_{2} \mathrm{CO}$ ?)

- What molecules are good tracers for outflows? (SiO?)

- What are the ionization levels in molecular cloud cores, in disks, and in winds and jets?

\section{References}

Adams, F.C., Lada, C.J., Shu, F.H. 1987, ApJ 312, 788

Adams, F.C., Lada, C.J., Shu, F.H. 1988, ApJ 326, 865

André, P., Ward-Thompson, D., Barsony, M. 1993, ApJ 406, 122

Arons, J. 1986, in Plasma Penetration into Magnetospheres, ed. N. Kylafis, J. Papamastorakis, \& J. Ventura (Crete Univ. Press, Iraklion), p. 115

Balbus, S.A., Hawley, J.F. 1991, ApJ 376, 214

Basu, S., Mouschovias, T. 1994, ApJ 432, 720

Beckwith, S.V.W., Sargent, A.,I., Chini, R.S., Güsten, R. 1990, AJ 99, 924

Bell, K.R., Lin, D.N.C. 1994, ApJ 427, 987

Benson, P.J., Myers, P.C. 1989, ApJS 71, 89

Bertout, C. 1989, ARAA 27, 351

Bertout, C., Basri, G., Bouvier, J. 1988, ApJ 330, 350

Blake, G.A., van Dishoeck, E.F., Jansen, D.J., Groesbeck, T.D., Mundy, L.G. 1994, ApJ 428,680

Briceño, C., Hartmann, L.W., Stauffer, J.R., Gagné, M., Stern, R.A., Caillault, J.-P. 1997, AJ, in press

Calvet, N., Patinõ, A., Magris, G., D'Alessio, P. 1991, ApJ 380, 617

Camenzind, M. 1990, in Reviews of Modern Astronomy 3, ed. G. Klare (Springer, Berlin), p. 259

Carr, J.S., Tokunaga, A.T., Najita, J., Shu, F.H., Glassgold, A.E. 1993, ApJ 411, L37

Cassen, P., Moosman, A. 1981, Icarus 48, 353

Chernin, L., Masson, C. 1995, ApJ 455, 182

Choi, M., Evans, N.J., Gregersen, E.M., Wang, Y. 1995, ApJ 448, 742

Cohen, M., Kuhi, L.V. 1979, ApJS 41, 743

Crutcher, R.M., Troland, T.H., Goodman, A.A., Heiles, C., Kazés, I., Myers, P.C. 1993, ApJ 407, 175 
De Boisanger, C., Helmich, F.P., Van Dishoeck, E.F. 1996, A\&A 310, 315.

Draine, B.T., Roberge, W.G., Dalgarno, A. 1983, ApJ 264, 485

Evans, N.J., 1991, in Frontiers of Stellar Evolution, ed. D.L. Lambert (Astron. Soc. Pacific, San Francisco), p. 45.

Fiedler, R.A., Mouschovias, T. 1993, ApJ 415, 680

Galli, D., Shu, F.H. 1993, ApJ 417, 220

Gammie, C.F. 1996, ApJ 457, 355

Hartigan, P., Edwards, S., Ghandour, L. 1995, ApJ 452, 736

Hartmann, L., Hewitt, R., Calvet, N. 1994, ApJ 426, 669

Hartmann, L., Kenyon, S.J., Hartigan, P. 1993, in Protostars and Planets III, ed. E.H. Levy \& J.I. Lunine (Univ. of Arizona Press, Tucson), p. 497

Hayashi, C., Nakazawa, K., Nakagawa, Y. 1985, in Protostars and Planets II, ed. D.C. Black \& M.S. Matthews (Univ. of Arizona Press, Tucson), p. 1100

Hayashi, M., Ohashi, N., Miyama, S.M. 1993, ApJ 418, L71

Kenyon, S.J., Hartmann, L. 1987, ApJ 323, 714

Kenyon, S.J., Calvet, N., Hartmann, L. 1993, ApJ 407, 219

Königl, A. 1992, ApJ 370, L39

Lada, C.J 1995, in Molecular Clouds and Star Formation, ed. C. Yuan \& J.H. You (World Scientific, Singapore), p. 1

Lada, C.J., Fich, M. 1996, ApJ 459, 638

Lada, E.A. 1992, ApJ 393, L25

Lada, E.A., DePoy, D.L., Evans, N.J., Gatley, I. 1991, ApJ 371, 171

Laughlin, G., Bodenheimer, P. 1993, ApJ 403, 303

Li, Z.-Y. 1996, ApJ 473, L873

Li, Z.-Y., Shu, F.H. 1996, ApJ 472, 211

Li, Z.-Y., Shu, F.H. 1997, ApJ 475, L237

Lin, D.N.C., Papaloizou, J. 1993, in Protostars and Planets III, ed. E. H. Levy \& J.I. Luninie (Univ. of Arizona Press, Tucson), p. 749

Lizano, S., Shu, F.H. 1989, ApJ 342, 834

Lynden-Bell, D., Pringle, J.E. 1974, MNRAS 168, 603

Masson, C., Chernin, L. 1992, ApJ 387, L47

Masson, C., Chernin, L. 1993, ApJ 414, 230

McKee, C.F. 1989, ApJ 345, 782

Mestel, L., Paris, R.B. 1979, MNRAS 187, 337

Mizuno, A., Onishi, T., Hayashi, M., Ohashi, N., Sunada, K., Hasegawa, T., Fukui, Y. 1994, Nature 368,71

Mouschovias, T. 1979, ApJ 228, 1599

Myers, P.C. 1995, in Molecular Clouds and Star Formation, ed. C. Yuan \& J.H. You (World Scientific, Singapore), p. 47

Myers, P.C., Benson, P.J. 1983, ApJ 266, 309

Myers, P.C., Fuller, G.A., Goodman, A.A., Benson, P.J. 1991, ApJ 376, 561

Nakano, T. 1979, PASJ 31, 697

Nakano, T., Umebayashi, T. 1980, PASJ 32, 613

Najita, J., Carr, J.S., Glassgold, A.E., Shu, F.H., Tokunaga, A.T. 1996, ApJ 462, 919

Najita, J.R., Shu, F.H. 1994, ApJ 429, 808

Ostriker, E.C., Shu, F.H. 1995, ApJ 447, 813

Ostriker, E., Shu, F.H., Adams, F.C. 1992, ApJ 399, 192

Palla, F., Stahler, S. 1992, ApJ 392, 667

Rucinski, S.M. 1985, AJ 90, 2321

Shu, F.H. 1977, ApJ 214, 488

Shu, F. H. 1983, ApJ 273, 202

Shu, F.H., Adams, F.C., Lizano, S. 1987, ARAA, 25, 23

Shu, F.H., Lizano, S., Ruden, S.P., Najita, J. 1988, ApJ 328, L19

Shu, F.H., Najita, J., Ostriker, E.C., Shang, H. 1995, ApJ 455, L155

Shu, F., Najita, J., Ostriker, E., Wilkin, F., Ruden, S., Lizano, S. 1994, ApJ 429, 781 
Shu, F.H., Ruden, S.P., Lada, C.J., Lizano, S. 1991, ApJ 370, L31

Shu, F.H., Terebey, S. 1984, in Cool Stars, Stellar Systems, and the Sun, ed. S. Baliunas \& L. Hartmann (Springer-Verlag, Berlin), p. 78

Stahler, S.W., Shu, F.H., Taam, R.E. 1980, ApJ 241, 637

Strom, K.M., Strom, S.E., Edwards, S., Cabrit, S., Skrutskie, M.F. 1989, AJ 97, 1451

Terebey, S., Shu, F.H., Cassen, P. 1984, ApJ 286, 529

Van Langevelde, H.J., van Dishoeck, E.F., Blake, G.A. 1994, ApJ 425, L45

Walter, F.M. 1986, ApJ 306, 573

Welch, W.J., Dreher, J.W., Jackson, J.M., Terebey, S., Vogel, S.N. 1987, Science 238, 1550

Whitney, B.A., Hartmann, L. 1993, ApJ 402, 605

Wright, M.C.H., Plambeck, R.L., Wilner, D. 1996, ApJ 469, 216

Yuan, C., Cassen, P. 1994, ApJ 437, 338

Zhou, S., Evans, N.J., Hayashi, Kömpe, C.M. Walmsley 1993, ApJ 404, 232 (Errata: 1994, ApJ 421, 854)

\section{Discussion}

Buckley: Wouldn't a better initial condition for collapse be the critical BonnerEbert isothermal sphere, which was shown in the simulations of Foster \& Chevalier to be unstable to collapse? This has only a moderate degree of central condensation, and a cloud evolving towards the singular isothermal sphere would presumably have to pass through this critical state. Also the models of Boss and his co-workers have shown that binary formation is severely inhibited when a rotating singular isothermal sphere is used as a starting point. Isn't the singular isothermal sphere therefore in conflict with the oberservations?

Shu: Direct numerical simulations show that the presence of magnetic fields delays the onset of gravitational instability until much higher central densities. Moreover, the motions generated by gravitational instability remain small until the core almost reaches the singular state. In such circumstances, when the density contrast between center and edge is extreme, the work of Foster \& Chevalier itself shows that the subsequent collapse (for $t>0$ ) would mimic closely that of the singular case. It is true that a high central concentration suppresses binary formation by the mechanims Boss considered. This does not imply, however, that binary formation cannot occur by some other route, via the fragmentation of disks, for example. Observationally, the situation is ambiguous. Tony Allen and I have shown that the presence of two accumulating protostars instead of one central one is typically very hard to distinguish in channel maps investigating models of infall.

Evans: You have stressed the toroidal configuration, but Phil Myers and others have argued that the cores in dark clouds are prolate. Do you have any thoughts on this question?

Shu: Myers et al. had two main arguments for clouds being prolate. 1. To account for the average observed flattening in projection of about $2: 1$, oblate spheroids would have to have intrinsic flattenings of 5:1 or more - too much for Myers et al. to be natural, so they argued clouds must be intrinsically prolate. For toroids, $\mathrm{Li}$ and $\mathrm{I}$ have shown that the intrinsic flattening needs to be much less to account for the observed ratio. The absence of matter above the poles helps enormously to make the column density contours look flatter at most angles of projection.

2. Many cores are flattened in the same direction as the filaments to which they 
belong are elongated. This seemed to Myers et al. to argue also for cores to be prolate. However, Nobeyama observations have shown that filaments are perpendicular to the ambient magnetic field, which is the same orientation as theory predicts for the flattening of magnetized molecular cloud cores. Thus, I believe the orientation of cloud cores with repect to filaments is not an argument for their being prolate. It has to be admitted however, that we have no clear understanding of molecular cloud filaments.

Walmsley: What is the essential difference in the physics of 'low mass' and 'high mass' star formation as you see it?

Shu: I don't think there is an 'intrinsic difference'. High-mass protostars evolve much more quickly, have much more ionizing radiation and do more damage to their surroundings once they form, but the effects probably form a continuum rather than a bimodal pattern of behavior. Two effects, however, limit the ability (at least, my ability) to calculate honest models. 1. The regions from which high mass stars are born are much more turbulent than the regions of low mass stars. Our understanding of interstellar turbulence is too primitive to allow its incorporation theoretically in any practical way other than as a source of pressure additional to thermal gas pressure. My group has advocated doing this too, but there may be important physical effects hidden by such a procedure. 2. High mass stars tend to be born in very crowded environments. The effects of neighbouring stars and cores may not be negligible. This again limits the theorist's ability to make relevant calculations and models.

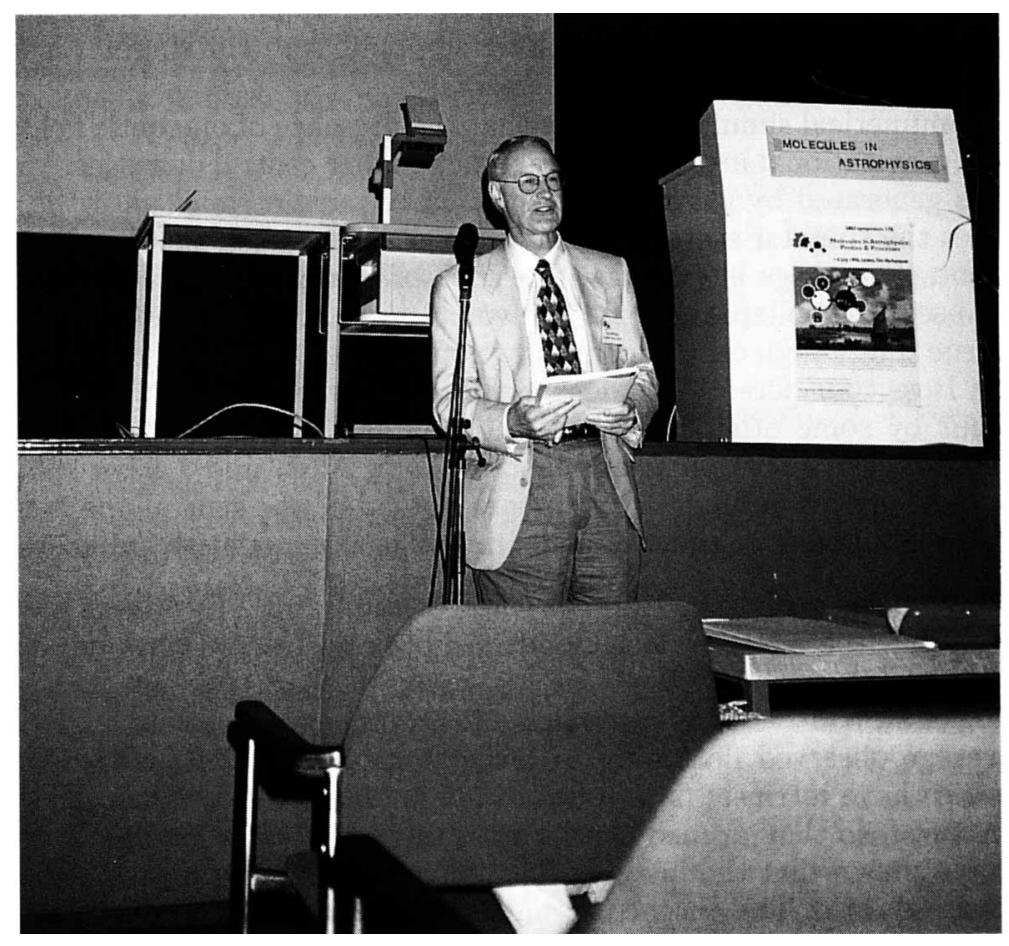

